\title{
Psoriasis localized to the glans penis in a 37-year-old man
}

\author{
Xiao-Jian Yao MM, Tang-De Zhang MD
}

— Cite as: CMAJ 2018 June 18;190:E747. doi: 10.1503/cmaj.180262

A 37-year-old man presented to the dermatology clinic with a seven-week history of pruritic, burning, scaly erythema on the glans penis. After the onset of symptoms, the patient did not engage in any sexual activity as he felt considerable discomfort and embarrassment. He was treated empirically with topical ketoconazole cream for two weeks without improvement. Physical examination showed redness and fine scaling on the glans penis
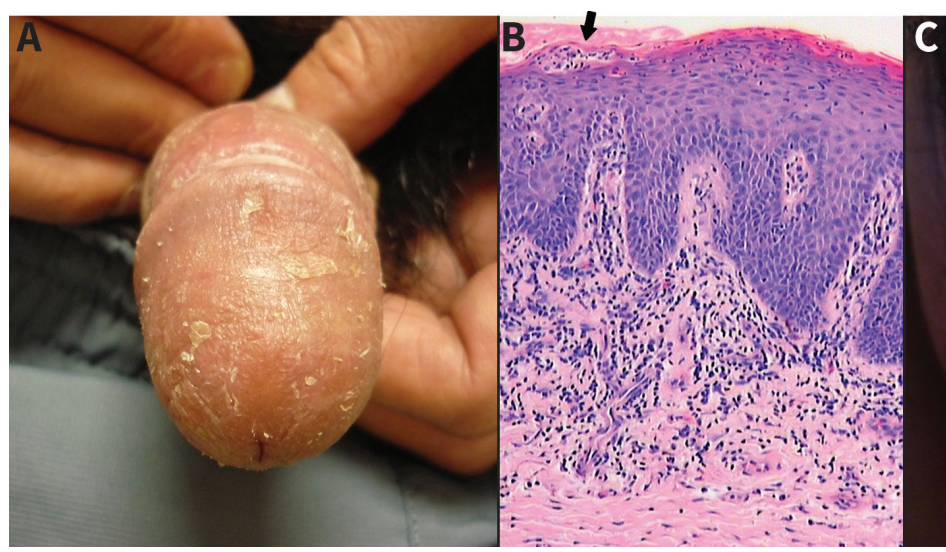

C

Figure 1: (A) Redness and fine scaling over the glans penis of a 37-year-old man. (B) Histopathologic image showing parakeratosis, Munro microabscesses (arrow) and acanthosis in the epidermis (hematoxylin and eosin stain, original magnification $\times 200$ ). (C) A substantial improvement of genital lesions after topical treatment with tacrolimus.
(Figure 1A). Fungal cultures of scrapings from the glans were negative. Skin biopsy showed parakeratosis, Munro microabscesses and acanthosis in the epidermis (Figure 1B), findings that were consistent with psoriasis vulgaris. The patient applied tacrolimus $0.1 \%$ ointment twice daily, resulting in resolution of the lesions after three weeks (Figure 1C). He then applied topical tacrolimus twice weekly for six months, during which the rash did not recur.

Up to $63 \%$ of patients with psoriasis vulgaris have a history of genital involvement, ${ }^{1}$ but psoriasis limited to the glans penis is uncommon, affecting only $2 \%-5 \%$ of patients with psoriasis. ${ }^{2}$ This distribution may affect quality of life and sexual activity. Patients may lack other signs of psoriasis, leading to misdiagnosis as other inflammatory diseases, such as dermatitis, candidiasis, plasma cell balanitis, lichen planus or lichen sclerosus. ${ }^{2}$

Glans skin is very thin and highly sensitive, which makes glans psoriasis difficult to treat. First-line topical treatment for psoriasis vulgaris includes potent corticosteroids, calcipotriol and tazarotene, ${ }^{3}$ but they are not the ideal options for glans psoriasis, especially for long-term use, because of their local adverse effects. Topical tacrolimus, a T cell-targeted immunosuppressant, has shown substantial efficacy and good tolerability in the treatment of inverse psoriasis that affects the face, genitals and intertriginous areas. ${ }^{4}$ Importantly, because topical tacrolimus can be applied for long periods without risk of local adverse effects, it is better suited to a patient with chronic or recurrent glans psoriasis. ${ }^{4}$

\section{References}

1. Ryan C, Sadlier M, De Vol E, et al. Genital psoriasis is associated with significant impairment in quality of life and sexual functioning. J Am Acad Dermatol 2015; 72:978-83.

2. Meeuwis KA, de Hullu JA, Massuger LF, et al. Genital psoriasis: a systematic literature review on this hidden skin disease. Acta Derm Venereol 2011;91:5-11.

3. Menter A, Korman NJ, Elmets CA, et al. Guidelines of care for the management of psoriasis and psoriatic arthritis. Section 3. Guidelines of care for the management and treatment of psoriasis with topical therapies. J Am Acad Dermatol 2009;60:643-59.

4. Malecic N, Young H. Tacrolimus for the management of psoriasis: clinical utility and place in therapy. Psoriasis (Auckl) 2016;6:153-63.
Competing interests: None declared.

This article has been peer reviewed.

The authors have obtained patient consent.
Affiliation: Department of Dermatology, Zhujiang Hospital, Southern Medical University, Guangzhou, China.

Correspondence to: Tang-De Zhang, tdgz2017@163.com 